Geometry $8 \mathcal{F}$ Topology

Volume 3 (1999) 369-396

Published: 26 October 1999

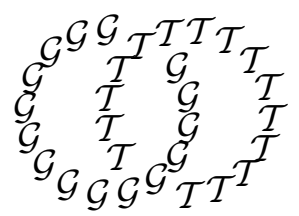

\title{
Circle-valued Morse theory and Reidemeister torsion
}

\author{
Michael Hutchings \\ YI-JEN LEE
}

Dept of Math, Stanford University, Stanford, CA 94305, USA

Dept of Math, Princeton University, Princeton, NJ 08544, USA

Email: hutching@math.stanford.edu and ylee@math.princeton.edu

\begin{abstract}
Let $X$ be a closed manifold with $\chi(X)=0$, and let $f: X \rightarrow S^{1}$ be a circlevalued Morse function. We define an invariant $I$ which counts closed orbits of the gradient of $f$, together with flow lines between the critical points. We show that our invariant equals a form of topological Reidemeister torsion defined by Turaev [28].

We proved a similar result in our previous paper [7], but the present paper refines this by separating closed orbits and flow lines according to their homology classes. (Previously we only considered their intersection numbers with a fixed level set.) The proof here is independent of the proof in [7], and also simpler.

Aside from its Morse-theoretic interest, this work is motivated by the fact that when $X$ is three-dimensional and $b_{1}(X)>0$, the invariant $I$ equals a counting invariant $I_{3}(X)$ which was conjectured in [7] to equal the Seiberg-Witten invariant of $X$. Our result, together with this conjecture, implies that the Seiberg-Witten invariant equals the Turaev torsion. This was conjectured by Turaev [28] and refines the theorem of Meng and Taubes [14].
\end{abstract}

AMS Classification numbers Primary: 57R70

Secondary: 53C07, 57R19, 58F09

Keywords: Morse-Novikov complex, Reidemeister torsion, Seiberg-Witten invariants

Proposed: Ralph Cohen

Received: 28 June 1999

Seconded: Robion Kirby, Steve Ferry

Accepted: 21 October 1999

\section{Copyright Geometry and Topology}




\section{Introduction}

Given a flow on a manifold, it is natural to ask how many closed orbits there are. It turns out that for some well-behaved flows, the numbers of closed orbits in different homology classes are related to the Reidemeister torsion of the underlying manifold. For example, Fried [2] defined a "twisted Lefschetz zeta function" counting closed orbits of certain nonsingular hyperbolic flows and showed that it equals a version of topological Reidemeister torsion, which is independent of the flow.

In this paper, we are interested in the gradient flow of a circle-valued Morse function. For singular flows such as this one, the zeta function is no longer invariant under deformation of the flow. It turns out that this lack of invariance can be fixed by considering the Novikov complex, which counts gradient flow lines between critical points. We will show that one can obtain a topological invariant by multiplying the zeta function by the Reidemeister torsion of the Novikov complex. We call the resulting invariant $I$.

In our previous work [7], we defined a weaker version of $I$ and showed that it equals a form of topological Reidemeister torsion. Later we received a preprint from Turaev [28] defining a refined version of the latter invariant, which we call "Turaev torsion" here. Along similar lines we can refine the Morse theoretic invariant in [7] to obtain the invariant $I$. The main result of this paper asserts that $I$ equals Turaev torsion.

Our previous methods are not quite sufficient to prove this refinement, so here we introduce a different and simpler approach. This paper is independent of [7], except that the latter paper defines certain compactifications in Morse theory which we use here, and also provides more background and context.

We now proceed to define our invariant $I$ more precisely and state our main theorem. We then describe the application to three-dimensional Seiberg-Witten theory. In section 2 we give some background definitions, and in section 3 we prove the main theorem. In section 4 we give more details on the relation to Seiberg-Witten theory.

\section{$1.1 \quad$ Statement of results}

The basic setup for this paper is as follows. Let $X$ be a closed connected oriented $n$-dimensional manifold. We assume throughout that $\chi(X)=0$, so that we can define Reidemeister torsion. Also, our result is most interesting when $b_{1}(X)>0$. 
Let $f: X \rightarrow S^{1}$. In order to consider the gradient flow of $f$, we endow $X$ with a Riemannian metric. We make the following assumptions:

Assumption $1.1 \quad$ (a) $f$ is a Morse function.

(b) The ascending and descending manifolds of the critical points of $f$ intersect transversely (see section 2.1).

(c) The closed orbits of the gradient $\nabla f$ are nondegenerate (see below).

A standard transversality argument shows that these assumptions hold if $f$ and the metric are generic.

A closed orbit is a nonconstant map $\gamma: S^{1} \rightarrow X$ with $\gamma^{\prime}(t)=-\lambda \nabla f$ for some $\lambda>0$. We declare two closed orbits to be equivalent if they differ by reparametrization. The period $p(\gamma)$ is the largest integer $p$ such that $\gamma$ factors through a $p$-fold covering $S^{1} \rightarrow S^{1}$. A closed orbit is nondegenerate if $\operatorname{det}(1-$ $d \phi(x)) \neq 0$, where $\phi$ is the $p^{\text {th }}$ return map at a point $x \in \gamma\left(S^{1}\right) \subset X$. If so, the Lefschetz sign $\epsilon(\gamma)$ is the sign of this determinant.

Notation 1.2 Let $H_{1}:=H_{1}(X)$. Let $\theta \in H^{1}(X ; \mathbb{Z})$ denote the pullback by $f$ of the "upward" generator of $H^{1}\left(S^{1} ; \mathbb{Z}\right)$.

Let $\Lambda=\operatorname{Nov}\left(H_{1},-\theta\right)$ denote the Novikov ring [17, 5], consisting of functions $H_{1} \rightarrow \mathbb{Z}$ that are finitely supported on the set $\left\{h \in H_{1} \mid-\theta(h) \leq C\right\}$ for each $C \in \mathbb{R}$. This ring has the obvious addition, and the convolution product. We denote a function $a: H_{1} \rightarrow \mathbb{Z}$ by the (possibly infinite) formal sum $\sum_{h \in H_{1}} a(h) \cdot h$.

Definition 1.3 $[2,20]$ We count closed orbits with the zeta function, which is a function $H_{1} \rightarrow \mathbb{Z}$ defined, in the above notation, by

$$
\zeta:=\exp \left(\sum_{\gamma \in \mathcal{O}} \frac{\epsilon(\gamma)}{p(\gamma)}[\gamma]\right)
$$

Here $\mathcal{O}$ denotes the set of closed orbits, and $[\gamma]:=\gamma_{*}\left[S^{1}\right]$ is the homology class of $\gamma$ in $H_{1}(X)$.

A compactness argument using Assumption 1.1, together with the observation that $-\theta([\gamma])>0$ for all $\gamma$, shows that $\zeta \in \Lambda \otimes \mathbb{Q}$.

We remark that there is also a product formula $[3,7,8]$

$$
\zeta=\prod_{\gamma \in \mathcal{I}}\left(1-(-1)^{i_{-}}[\gamma]\right)^{-(-1)^{i_{0}}} .
$$


Here $\mathcal{I}$ denotes the set of irreducible (period 1) closed orbits, and $i_{-}(\gamma)$ and $i_{0}(\gamma)$ denote the numbers of real eigenvalues of the return map in the intervals $(-\infty,-1)$ and $(-1,1)$, respectively. Equation (2) shows that in fact $\zeta \in \Lambda$, ie, $\zeta$ has integer coefficients. A third formula for the zeta function, in terms of fixed points of return maps, is given in equation (13).

We now introduce a notion of topological Reidemeister torsion following Turaev [28], and an analogous notion of Morse-theoretic torsion. Detailed definitions are given in section 2.3 .

Let $\tilde{X}$ denote the universal (connected) abelian cover of $X$, whose automorphism group is $H_{1}(X)$. A smooth triangulation of $X$ lifts to $\tilde{X}$ and gives rise to a chain complex $C_{*}(\tilde{X})$ over $\mathbb{Z}\left[H_{1}\right]$. The Reidemeister torsion of this complex is an element of $Q\left(\mathbb{Z}\left[H_{1}\right]\right) / \pm H_{1}$, where $Q(R)$ denotes the total quotient ring of $R$. The $\pm H_{1}$ ambiguity arises because the Reidemeister torsion depends on a choice of ordered basis.

Turaev [27] showed that the $H_{1}$ ambiguity can be resolved by the choice of an "Euler structure". The space $\operatorname{Eul}(X)$ of Euler structures is a natural affine space over $H_{1}(X)$, reviewed in section 2.2. One can also resolve the sign ambiguity by choosing a homology orientation of $X$, ie, an orientation $o$ of $\bigoplus_{i} H_{i}(X ; \mathbb{Q})$ (see $\left.[26]\right)$. We can then define the Turaev torsion

$$
\tau(X ; o): \operatorname{Eul}(X) \rightarrow Q\left(\mathbb{Z}\left[H_{1}\right]\right) .
$$

This is an $H_{1}$-equivariant map which does not depend on the triangulation. We write $\tau(X):= \pm \tau(X, o)$; this is an $H_{1}$-equivariant map $\operatorname{Eul}(X) \rightarrow Q\left(\mathbb{Z}\left[H_{1}\right]\right) / \pm 1$.

Example 1.4 If $X$ is the 3-manifold obtained by zero surgery on a knot $K \subset S^{3}$, then for a suitable Euler structure $\xi$,

$$
\tau(X)(\xi)=\frac{\operatorname{Alex}(K)}{(1-t)^{2}}
$$

where $\operatorname{Alex}(K) \in \mathbb{Z}\left[t, t^{-1}\right] / \pm 1$ is the Alexander polynomial of $K$ and $t$ is a generator of $H_{1}(X) \simeq \mathbb{Z}$.

On the Morse theory side, the Novikov complex $C N_{*}$ is a chain complex over the Novikov ring $\Lambda$, whose chains are generated by critical points of the pullback of $f$ to $\tilde{X}$, and whose boundary operator counts gradient flow lines between critical points (see section 2.1). We can similarly define the Morse-theoretic torsion

$$
\tau\left(C N_{*}\right): \operatorname{Eul}(X) \rightarrow Q(\Lambda) / \pm 1
$$


Definition 1.5 Define $I: \operatorname{Eul}(X) \rightarrow Q(\Lambda) / \pm 1$ to be the product of the zeta function and the Morse-theoretic torsion:

$$
I:=\zeta \cdot \tau\left(C N_{*}\right)
$$

Theorem 1.6 The Morse theory invariant $I$ is independent of the metric and depends only on the homotopy class of $f$, ie the cohomology class $\theta$.

One can prove this a priori; see [7] for the rough idea and [6] for the details. Although this may help define related invariants in other contexts, in the present context it is easier to compute $I$ directly, which will prove Theorem $1.6 a$ posteriori. That is what we will do in this paper.

Theorem 1.7 (Main theorem) Our Morse theory invariant $I$ is equal to the topological torsion:

$$
I=i(\tau(X))
$$

as maps $\operatorname{Eul}(X) \rightarrow Q(\Lambda) / \pm 1$.

Here $i: Q\left(\mathbb{Z}\left[H_{1}\right]\right) \rightarrow Q(\Lambda)$ is induced by the inclusion $\mathbb{Z}\left[H_{1}\right] \rightarrow \Lambda$.

Remarks 1.8 (1) In the extreme case when there are no critical points, $X$ is a mapping torus and this theorem reduces to an equivariant version of the Lefschetz fixed point theorem, cf $[15,3]$.

(2) The extreme case when $\theta=0$, so that $f$ lifts to a real-valued Morse function, is also essentially classical (cf [16]), and we treat it in section 2.4. In this case the Morse-theoretic torsion is a topological invariant; lack of invariance and existence of closed orbits arise simultaneously when we pass from realvalued to circle-valued Morse theory.

(3) The class $\theta \in H^{1}(X)$, regarded as a map $H_{1}(X) \rightarrow \mathbb{Z}$, induces a map $\Lambda \rightarrow \mathbb{Z}((t))$ sending $h \mapsto t^{\theta(h)}$. This in turn induces a partially defined map $Q(\Lambda) \rightarrow \mathbb{Q}((t))$. One can deduce the main result of our previous paper [7] by applying this map to Theorem 1.7.

(4) The statement of Theorem 1.7 makes sense when $d f$ is replaced by a generic closed 1 -form $\mu$ and the Novikov ring is graded by $-[\mu]$. It seems possible to extend Theorem 1.7 to this case by approximating $\mu$ by closed 1 -forms in rational cohomology classes, to which Theorem 1.7 applies. Another proof for closed 1-forms is given in [6] by first proving Theorem 1.6 for closed 1-forms, and then using this to reduce to the real-valued case. 
(5) Some previous papers, such as [19], studied the torsion of the Novikov complex (or the Whitehead torsion, which is sharper but only defined "relatively" unless the Novikov complex is acyclic), without considering the zeta function. In this case one can still obtain a topological invariant by modding out by units in the Novikov ring with leading coefficient 1 . This is useful for understanding the obstructions to the existence of nonvanishing closed 1 -forms [10, 4]. However, the extra information in the zeta function is important for the connection with Seiberg-Witten theory below.

(6) A homology orientation of $X$ can apparently remove the sign ambiguity in $\tau\left(C N_{*}\right)$. However we have not checked if Theorem 1.7 holds with the sign ambiguity removed this way.

\subsection{Application to Seiberg-Witten theory}

We now consider the special case when $\operatorname{dim}(X)=3$ and $b_{1}(X)>0$. Let $\operatorname{Spin}^{c}(X)$ denote the set of spin-c structures on $X$. Given a homology orientation $o$, the Seiberg-Witten invariant of $X$ is a function

$$
\mathrm{SW}_{X, o}: \operatorname{Spin}^{c}(X) \rightarrow \mathbb{Z}
$$

which counts $\mathbb{R}$-invariant solutions to the Seiberg-Witten equations on $X \times \mathbb{R}$, modulo gauge equivalence. (See eg $[13,14,18]$. )

Taubes [24] has shown that the SW invariant of a symplectic four-manifold equals a "Gromov invariant" counting pseudoholomorphic curves. In [7] we proposed that using similar analysis, one might be able to show that the SW invariant of a 3-manifold is equal to a Morse theory invariant

$$
I_{3}: \operatorname{Spin}^{c}(X) \rightarrow \mathbb{Z} .
$$

The invariant $I_{3}$ counts certain unions of closed orbits and flow lines of the gradient vector field of a Morse function $f: X \rightarrow S^{1}$ with no index 0 or 3 critical points. We review the definition of $I_{3}$ in section 4 .

Conjecture 1.9 [7] The Seiberg-Witten invariant agrees with our Morse theory invariant:

$$
\mathrm{SW}_{X, o}= \pm I_{3}
$$

(When $b_{1}(X)=1$, the SW invariant also depends on a choice of "chamber", and in this conjecture we use the chamber determined by $r * d f$ for $r>>0$.) 
Remark 1.10 If $f$ has no critical points, and if we arrange for $d f$ to be harmonic, then this conjecture is a corollary of Taubes' theorem [24] applied to the symplectic four-manifold $\left(X \times S^{1}, d f \wedge d s+*_{X} d f\right)$. Here $s$ denotes the $S^{1}$ coordinate. The idea is that for a suitable homology orientation, if $\mathfrak{s} \in \operatorname{Spin}^{c}(X)$, then

$$
\mathrm{SW}_{X, o}(\mathfrak{s})=\mathrm{SW}_{X \times S^{1}}\left(\pi^{*} \mathfrak{s}\right)=\operatorname{Gr}_{X \times S^{1}}\left(\pi^{*} \mathfrak{s}\right)=I_{3}(\mathfrak{s}) .
$$

The first equality expresses the fact that all solutions to the SW equations on $X \times S^{1}$ are $S^{1}$-invariant; see [18] for details of this equality. The second equality is Taubes' theorem; here $\operatorname{Gr}_{X \times S^{1}}\left(\pi^{*} \mathfrak{s}\right)$ counts, in the sense of [25], pseudoholomorphic curves in a certain $S^{1}$-invariant homology class in $X \times S^{1}$. An energy argument shows that for a suitable almost complex structure, every such curve is a union of closed orbits of $\nabla f$ crossed with $S^{1}$. This leads to the third equality ( $\mathrm{cf}\left[8\right.$, Thm. 0.1]), using the fact that $I_{3}$ is a reparametrization of the zeta function in this case.

Salamon has proved a statement equivalent to Conjecture 1.9 in this case using a different method [22].

In another direction, Turaev [28] conjectured a combinatorial formula for the Seiberg-Witten invariant as follows. If $\operatorname{dim}(X)=3$ and $b_{1}(X)>1$, then for an Euler structure $\xi$, the torsion $\tau(X ; o)(\xi)$ is actually in the group ring $\mathbb{Z}\left[H_{1}\right]$. If $b_{1}=1$, then $i(\tau(X ; o)(\xi)) \in \Lambda$, rather than in the quotient ring. Given a homology orientation $o$, one can then define a map

$$
\begin{aligned}
T(X ; o): \operatorname{Eul}(X) & \longrightarrow \mathbb{Z}, \\
\xi & \longmapsto i(\tau(X ; o)(\xi))(0) .
\end{aligned}
$$

where $(0)$ indicates evaluation on $0 \in H_{1}$. (This depends on the sign of $[d f]$ when $b_{1}=1$.) There is also a natural isomorphism $\imath: \operatorname{Spin}^{c}(X) \rightarrow \operatorname{Eul}(X)$ ([27], see section 4.3).

Conjecture 1.11 (Turaev [28]) The Seiberg-Witten invariant agrees with the Turaev torsion:

$$
\mathrm{SW}_{X, o}=T(X ; o) \circ \imath: \operatorname{Spin}^{c}(X) \rightarrow \mathbb{Z} .
$$

This statement is a refinement of the theorem of Meng and Taubes [14], which gives an "averaged" version of this equivalence, in which one sums over spin-c structures that differ by torsion elements of $H^{2}(X ; \mathbb{Z})$.

The invariant $I_{3}$ turns out to be a reparametrization of the more general invariant $I$. Thus we can apply Theorem 1.7 to prove: 
Theorem 1.12 Conjecture 1.9 is equivalent to Conjecture 1.11 (modulo signs).

The detailed proof is given in section 4 .

Update (1) Three days after the first version of this paper was posted on the internet, a preprint by Pajitnov [20] appeared, giving a result similar to Theorem 1.7, using Whitehead torsion.

(2) Turaev [29] has shown how to refine the methods of Meng and Taubes to prove Conjecture 1.11, modulo signs. Together with our results, this indirectly proves Conjecture 1.9. However, one might still desire a direct analytic proof. The following is a summary of the situation:

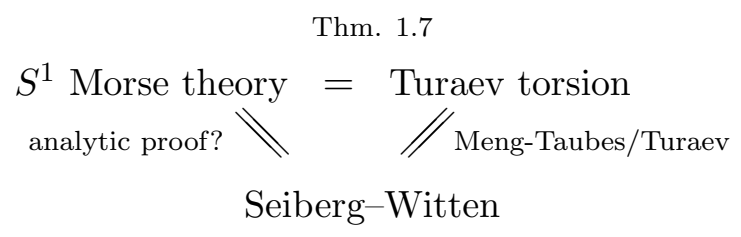

Acknowledgments This paper would not exist were it not for Taubes' work on Seiberg-Witten and Gromov invariants. We thank him for sharing his ideas generously. We also thank R Bott, R Forman, and D Salamon for helpful conversations.

\section{Background}

We now give some necessary background. Section 2.1 reviews the definition of the Novikov complex, which counts gradient flow lines between critical points. Section 2.2 reviews Turaev's Euler structures, which are needed for the most refined version of Reidemeister torsion. Section 2.3 gives the precise definitions of the versions of Reidemeister torsion that we use. Finally, section 2.4 proves Theorem 1.7 for real-valued Morse functions, as a warmup for some of the arguments in section 3 .

\subsection{The Novikov complex}

We begin with some standard definitions from Morse theory. If $p$ is a critical point in $X$ of $f$ or in $\tilde{X}$ of the pullback of $f$, the descending manifold $\mathcal{D}(p)$ is the set of all points $x$ such that upward gradient flow starting at $x$ converges 
to $p$. Similarly the ascending manifold $\mathcal{A}(p)$ is the set of all points from which downward gradient flow converges to $p$. If ind $(p)=i$, then $\mathcal{D}(p)$ and $\mathcal{A}(p)$ are embedded open balls of dimensions $i$ and $n-i$ respectively.

The Novikov complex $\left(C N_{*}, \partial^{f}\right)$ is defined as follows. On $\tilde{X}$, we can lift our Morse function to a map $\tilde{f}: \tilde{X} \rightarrow \mathbb{R}$. Let $C N_{i}$ denote the set of (possibly infinite) linear combinations $\alpha$ of critical points of index $i$ in $\tilde{X}$, such that for each $C \in \mathbb{R}$, the sum $\alpha$ contains only finitely many critical points $p \in \tilde{X}$ with $\tilde{f}(p)>C$. The action of $H_{1}$ on the critical points by covering transformations induces an action of the Novikov ring $\Lambda$ on $C N_{i}$. In fact $C N_{i}$ is a free $\Lambda_{-}$ module; one can specify a basis for $C N_{*}$ by choosing a lift of each critical point in $X$ to $\tilde{X}$.

We define $\partial^{f}: C N_{i} \rightarrow C N_{i-1}$ as follows. If $p, q \in \tilde{X}$ are critical points of index $i$ and $i-1$ respectively, let $\left\langle\partial^{f} p, q\right\rangle$ denote the signed number of gradient flow lines from $p$ to $q$. If $p$ is a critical point of index $i$, define

$$
\partial^{f} p:=\sum_{q}\left\langle\partial^{f} p, q\right\rangle q
$$

where the sum is over all critical points $q \in \tilde{X}$ of index $i-1$. We count flow lines using the sign conventions from [7]. These conventions are chosen so that $\left(\partial^{f}\right)^{2}=0$ and so that equation (16) holds.

Theorem 2.1 (Novikov) The homology of the Novikov complex is naturally isomorphic to the homology of the "half-infinite" chains in $\tilde{X}$ :

$$
H_{*}\left(C N_{*}, \partial^{f}\right) \simeq H_{*}\left(C_{*}(\tilde{X}) \otimes \Lambda\right) .
$$

See eg $[17,19,21,7]$. For example, if $X=S^{1}$ and $f: S^{1} \rightarrow S^{1}$ has nonzero degree, then the homology of the Novikov complex vanishes.

\section{$2.2 \quad$ Euler structures}

We now discuss three different notions of "Euler structure" and how they relate. One can ignore this material at the expense of allowing an $H_{1}$ ambiguity in Reidemeister torsion.

Definition 2.2 (Turaev [27]) If $X$ is a closed smooth manifold with $\chi(X)=$ 0 and $n=\operatorname{dim}(X)>1$, a smooth Euler structure on $X$ is a nonsingular vector field on $X$, where two such vector fields are declared equivalent if their restrictions to the complement of a ball in $X$ are homotopic through nonsingular vector fields. We let $\operatorname{Eul}(X)$ denote the space of smooth Euler structures. 
By obstruction theory, $\operatorname{Eul}(X)$ is an affine space over $H^{n-1}\left(X ; \pi_{n-1}\left(S^{n-1}\right)\right)=$ $H_{1}(X)$. (It is nonempty since $\chi(X)=0$.)

The following alternate definition of smooth Euler structures is useful for Morse theory, and also works well when $n=1$. If $v$ is a vector field on $X$ with nondegenerate zeroes, let $H_{1}(X, v)$ denote the set of homology classes of 1chains $\gamma \in X$ with $\partial \gamma=v^{-1}(0)$, where the points in $v^{-1}(0)$ are oriented in the standard way. The set $H_{1}(X, v)$ is a subset of the relative homology $H_{1}\left(X, v^{-1}(0)\right)$ and is an affine space over $H_{1}(X)$.

Definition 2.3 One can show by a cobordism argument that the spaces $H_{1}(X, v)$ for different $v$ 's are canonically isomorphic to each other, and hence to a single space. We call this space $\operatorname{Eul}(X)$. We let $i_{v}: H_{1}(X, v) \rightarrow \operatorname{Eul}(X)$ denote the canonical isomorphism.

If $n>1$, we can go from Definition 2.3 to Definition 2.2 as follows. Given $\gamma \in H_{1}(X, v)$, we can represent $\gamma$ by disjoint paths connecting the zeroes of $v$ in pairs. We then construct a nonsingular vector field by cancelling the zeroes of $v$ in a neighborhood of $\gamma$. (If $v$ has no zeroes, we send $0 \in H_{1}(X)=H_{1}(X, v)$ to the Euler structure represented by $v$ and extend equivariantly.)

Definition 2.4 [27] Let $(X, T)$ be a finite connected CW-complex with cells $\left\{\sigma_{i}\right\}$. ( $X$ denotes the underlying topological space; $T$ denotes the cell structure.) A combinatorial Euler structure on $(X, T)$ is a choice of a lift of each cell to the universal abelian cover $\tilde{X}$, where two such sets of lifts $\left\{\tilde{\sigma}_{i}\right\}$ and $\left\{h_{i} \tilde{\sigma}_{i}\right\}$, with $h_{i} \in H_{1}(X)=\operatorname{Aut}(\tilde{X})$, are considered equivalent if $\sum_{i}(-1)^{\operatorname{dim}\left(\sigma_{i}\right)} h_{i}=0$.

We let $\operatorname{Eul}(X, T)$ denote the space of combinatorial Euler structures of the CW-complex $(X, T)$. This is clearly an affine space over $H_{1}(X)$.

Note that if $\bar{T}$ is a refinement of the cell-structure $T$ with cells $\left\{\tau_{j}\right\}$, then there is a canonical isomorphism $\operatorname{Eul}(X, T) \rightarrow \operatorname{Eul}(X, \bar{T})$ sending $\left\{\tilde{\sigma}_{i}\right\}$ to $\left\{\tilde{\tau}_{j}\right\}$, where $\tilde{\tau}_{j} \subset \tilde{\sigma}_{i}$ if $\tau_{j} \subset \sigma_{i}$.

Lemma 2.5 [27] If $X$ is a closed smooth manifold with a smooth triangulation $T$, then there is a natural isomorphism between the spaces of smooth and combinatorial Euler structures:

$$
\operatorname{Eul}(X) \simeq \operatorname{Eul}(X, T) .
$$


The idea is that there is a natural vector field on each simplex with a zero at the center of each face and which points into the simplex near the boundary. These piece together to give a continuous vector field on $X$. We can perturb this to a smooth vector field $v_{T}$ with a nondegenerate zero of sign $(-1)^{i}$ in the center of each $i$-simplex. Then a smooth Euler structure $\xi$ can be represented by a chain $\gamma$ consisting of paths connecting the zeroes in pairs, with $[\gamma]=i_{v_{T}}^{-1}(\xi) \in$ $H_{1}\left(X, v_{T}\right)$. We can lift the chain $\gamma$ to $\tilde{X}$, and the induced lifts of its endpoints determine a combinatorial Euler structure.

\section{$2.3 \quad$ Reidemeister torsion}

We now review the definition of Reidemeister torsion of certain chain complexes. We then use this algebra to define Reidemeister torsion for the two geometric complexes we are interested in.

\subsubsection{Algebra}

Let $\left(C_{*}, \partial\right)$ be a finite complex of finite dimensional vector spaces over a field $F$. The standard short exact sequences $0 \rightarrow Z_{i} \rightarrow C_{i} \rightarrow B_{i-1} \rightarrow 0$ and $0 \rightarrow B_{i} \rightarrow$ $Z_{i} \rightarrow H_{i} \rightarrow 0$ induce canonical isomorphisms $\operatorname{det}\left(C_{i}\right)=\operatorname{det}\left(Z_{i}\right) \otimes \operatorname{det}\left(B_{i-1}\right)$ and $\operatorname{det}\left(Z_{i}\right)=\operatorname{det}\left(B_{i}\right) \otimes \operatorname{det}\left(H_{i}\right)$, where 'det' denotes top exterior power. Combining these isomorphisms gives an isomorphism

$$
\Phi: \bigotimes_{i} \operatorname{det}\left(C_{i}\right)^{(-1)^{i}} \rightarrow \bigotimes_{i} \operatorname{det}\left(H_{i}\right)^{(-1)^{i}}
$$

Let $e$ be an ordered basis for $C_{*}$, ie, an ordered basis $e_{i}$ for each $C_{i}$. Let $h$ be an ordered basis for $H_{*}$. Let $[e] \in \bigotimes_{i} \operatorname{det}\left(C_{i}\right)^{(-1)^{i}}$ and $[h] \in \bigotimes_{i} \operatorname{det}\left(H_{i}\right)^{(-1)^{i}}$ denote the resulting volume forms.

Definition 2.6 We define the Reidemeister torsion

$$
\hat{\tau}\left(C_{*}, e, h\right):=\Phi([e]) /[h] \in F^{\times} .
$$

We also define

$$
\tau\left(C_{*}, e\right):=\left\{\begin{array}{cl}
\hat{\tau}\left(C_{*}, e, 1\right) & \text { if } H_{*}=0 \\
0 & \text { otherwise }
\end{array}\right.
$$

Usually we will be interested in $\tau$ rather than $\hat{\tau}$. In practice, we can compute the torsion $\tau$ in terms of an alternating product of determinants as follows. 
Lemma 2.7 If $H_{*}=0$, we can find a decomposition $C_{*}=A_{*} \oplus B_{*}$ such that (i) $A_{i}$ and $B_{i}$ are spanned by subbases of the basis $e_{i}$, and (ii) the map

$$
\left.\pi_{B_{i-1}} \circ \partial\right|_{A_{i}}: A_{i} \rightarrow B_{i-1}
$$

(which we abbreviate by $\partial: A_{i} \rightarrow B_{i-1}$ ) is an isomorphism. Then

$$
\tau\left(C_{*}, e\right):= \pm \prod_{i} \operatorname{det}\left(\partial: A_{i} \rightarrow B_{i-1}\right)^{(-1)^{i}} .
$$

Here the determinants are computed using the subbases of $e$.

We now extend the definition of torsion to complexes over certain rings which might not be fields.

Definition 2.8 [28] Let $R$ be a ring, and assume that its total quotient ring (denoted by $Q(R)$ ) is a finite sum of fields, $Q(R)=\oplus_{j} F_{j}$. Let $\left(C_{*}, \partial\right)$ be a finite complex of finitely generated free $R$-modules with an ordered basis $e$. We define

$$
\tau\left(C_{*}, e\right):=\sum_{j} \tau\left(C_{*} \otimes_{R} F_{j}, e \otimes 1\right) \in \bigoplus_{j} F_{j}=Q(R) .
$$

In this case $H_{*}(C)$ might not be free, in which case it does not have a basis in the usual sense. However in this paper we call a set $h:=\left\{h_{j}\right\}$ a "basis" for $H_{*}(C)$ when $h_{j}$ is a basis for $H_{*}\left(C_{*} \otimes_{R} F_{j}\right)$ for each $j$. Given $h=\left\{h_{j}\right\}$, we define

$$
\hat{\tau}\left(C_{*}, e, h\right):=\sum_{j} \hat{\tau}\left(C_{*} \otimes_{R} F_{j}, e \otimes 1, h_{j}\right) \in Q(R)^{\times} .
$$

Example 2.9 If $0 \rightarrow C_{2} \stackrel{\partial}{\rightarrow} C_{1} \rightarrow 0$ is a 2-term complex with $C_{1}=C_{2}$, and if $e$ is a basis which is identical on $C_{1}$ and $C_{2}$, then

$$
\tau\left(C_{*}, e\right)=\operatorname{det}(\partial) \text {. }
$$

We are interested in the rings $\mathbb{Z}\left[H_{1}\right]$ and $\Lambda$. Their quotients are finite sums of fields (see eg [28]), and these decompositions are compatible with the inclusion $\mathbb{Z}\left[H_{1}\right] \rightarrow \Lambda$.

In section 3.2, we will need the following product formula for torsion. Let $R$ be a ring such that $Q(R)$ is a finite sum of fields $F_{j}$. Let $0 \rightarrow C_{*} \rightarrow C_{*}^{\prime} \rightarrow C_{*}^{\prime \prime} \rightarrow 0$ be a short exact sequence of finite complexes of finitely generated free $R$-modules. Let $e, e^{\prime}, e^{\prime \prime}$ be bases for $C_{*}, C_{*}^{\prime}, C_{*}^{\prime \prime}$ compatible with the exact sequence. Let $h, h^{\prime}, h^{\prime \prime}$ be bases for the homology as in Definition 2.8. Let $L_{*}$ denote the long exact sequence in homology, regarded as an acyclic chain complex, and let $b$ denote the basis for $L_{*}$ obtained by combining $h, h^{\prime}, h^{\prime \prime}$. 
Lemma 2.10 We have the following product formula for torsion:

$$
\hat{\tau}\left(C^{\prime}, e^{\prime}, h^{\prime}\right)=\hat{\tau}(C, e, h) \hat{\tau}\left(C^{\prime \prime}, e^{\prime \prime}, h^{\prime \prime}\right) \tau\left(L_{*}, b\right) .
$$

Proof This follows from [16].

\subsubsection{Geometry}

We now define the Turaev torsion (3). In the notation of Definition 2.4, let $(X, T)$ be a finite connected $\mathrm{CW}$-complex with universal abelian cover $\tilde{X}$. Lifing the cells gives a chain complex $C_{*}(\tilde{X}, T)$ over $\mathbb{Z}\left[H_{1}(X)\right]$. A combinatorial Euler structure $\xi$ determines a set of lifts of each cell to $\tilde{X}$, up to equivalence. Choose one set of lifts; this gives a basis for $C_{*}(\tilde{X}, T)$. A homology orientation $o$ determines an orientation of this basis, via the isomorphism (5) applied to $C_{*}(X, T)$. Let $e(\xi, o)$ denote the resulting ordered basis. We define the combinatorial Turaev torsion $\tau(X, T ; o)$ to be the $H_{1}$-equivariant map $\operatorname{Eul}(X, T) \rightarrow Q\left(\mathbb{Z}\left[H_{1}\right]\right)$ given by

$$
\tau(X, T ; o)(\xi):=\tau\left(C_{*}(\tilde{X}, T), e(\xi, o)\right) \in Q\left(\mathbb{Z}\left[H_{1}\right]\right) .
$$

Note that the right hand side of this equation does not depend on the choice of a set of lifts. Furthermore, $\tau(X, T ; o)=\tau(X, \bar{T} ; o)$ under the canonical isomorphism $\operatorname{Eul}(X, T) \rightarrow \operatorname{Eul}(X, \bar{T})$, if $\bar{T}$ is a refinement of $T$.

Definition 2.11 Let $X$ be a closed connected smooth manifold with $\chi(X)=$ 0 , with a smooth Euler structure $\xi \in \operatorname{Eul}(X)$ and a homology orientation $o$. Choose a smooth triangulation $T$ of $X$. Let $\xi_{T} \in \operatorname{Eul}(X, T)$ denote the combinatorial Euler structure equivalent to $\xi$ via Lemma 2.5. We define the Turaev torsion

$$
\tau(X ; o)(\xi):=\tau(X, T ; o)\left(\xi_{T}\right) .
$$

The results of [27] show that the Turaev torsion does not depend on the choice of smooth triangulation $T$.

We now define the Morse theoretic torsion (4). A smooth Euler structure $\xi$ can be represented by a chain $\gamma$ connecting the critical points of $f$ in pairs, with $[\gamma]=i_{\nabla f}^{-1}(\xi) \in H_{1}(X, \nabla f)$. We can lift $\gamma$ to $\tilde{X}$, and the induced lifts of the endpoints determine a basis $e(\xi)$ for $C N_{*}$.

Definition 2.12 We define the Morse theoretic torsion $\tau\left(C N_{*}\right): \operatorname{Eul}(X) \rightarrow$ $Q(\Lambda) / \pm 1$ by

$$
\tau\left(C N_{*}\right)(\xi):=\tau\left(C N_{*}, e(\xi)\right) .
$$


The map $\tau\left(C N_{*}\right)$ is $H_{1}$-equivariant, and again does not depend on the choice of lifting. There is a sign ambiguity because the basis $e(\xi)$ is unordered. In the special case when $f$ has no critical points, we define $\tau\left(C N_{*}\right)$ to be the $H_{1}$ equivariant map such that $\tau\left(C N_{*}\right)(\xi)=1$ for the smooth Euler structure $\xi$ represented by $\nabla f$.

In the future, we call two bases for $C_{*}(\tilde{X}, T)$ or $C N_{*}$ equivalent if they correspond to the same Euler structure $\xi$.

\subsection{The real-valued case}

Before proceeding more deeply into circle-valued Morse theory, it will be useful to prove the main theorem for real-valued Morse functions.

Lemma 2.13 Theorem 1.7 holds when $f: X \rightarrow S^{1}$ lifts to a real-valued Morse function $X \rightarrow \mathbb{R}$.

Proof In this case $\zeta=1$, so we just need to check that the Morse theoretic and topological torsions agree. This is essentially classical (cf [16]), except for the identification of the bases determined by an Euler structure.

If $\xi$ is an Euler structure, then the bifurcation analysis in [11] shows that $\tau\left(C N_{*}\right)(\xi)$ is independent of the real-valued Morse function and the metric. It is not hard to check that the Euler structures work out at each stage. In [11] it is assumed that the metric has a standard form near the critical points, but this can be arranged by a perturbation which does not affect the Novikov complex.

Now let $T$ be a smooth triangulation of $X$. We can apparently perturb the vector field $V_{T}$ of section 2.2 so that it is the gradient of a Morse function $F: X \rightarrow \mathbb{R}$ with respect to some metric. In this case, the Novikov complex $\left(C N_{*}, \partial^{F}\right)$ is identical to the chain complex $\left(C_{*}(\tilde{X} ; T), \partial\right)$. Moreover the bases determined by $\xi$ agree. Thus the Morse-theoretic and topological torsion are equal.

\section{Proof of the main theorem}

We will now prove the main theorem as follows. In section 3.1 we prepare for the computation of torsion by constructing a cell complex $X^{\prime}$ which "approximates" $X$ and is adapted to the vector field $\nabla f$. In section 3.2 we prove a technical 
lemma (Lemma 3.6) asserting that $X$ and $X^{\prime}$ have the same Reidemeister torsion. The heart of the proof is in section 3.3 and section 3.4, where we determine the torsion of $X^{\prime}$ by a short computation, and then interpret the answer geometrically to recover the invariant $I$.

\subsection{The cell complex $X^{\prime}$}

Assume $0 \in S^{1}=\mathbb{R} / \mathbb{Z}$ is a regular value of $f$ (by composing $f$ with a rotation if necessary). Let $\Sigma:=f^{-1}(0)$. Let $Y$ be the compact manifold with boundary obtained by cutting $X$ along $\Sigma$. We can write $\partial Y=\Sigma_{1} \sqcup \Sigma_{0}$, where $\Sigma_{i}$ is canonically isomorphic to $\Sigma$, and $-\nabla f$ points inward along $\Sigma_{1}$.

We give $Y$ a cell decomposition as follows. Let $T_{1}$ be a smooth triangulation of $\Sigma_{1}$ such that each simplex is transverse to the ascending manifolds of the critical points in $Y$. If $p \in Y$ is a critical point, let $\mathcal{D}_{0}(p)$ denote the descending manifold of $p$ in $Y$. If $\sigma \in T_{1}$ is a simplex, let $\mathcal{F}(\sigma)$ denote the set of all $y \in Y$ such that upward gradient flow starting at $y$ hits $\sigma$. Choose a cell decomposition $T_{0}$ of $\Sigma_{0}$, such that the intersections with $\Sigma_{0}$ of $\mathcal{D}_{0}(p)$ and $\mathcal{F}(\sigma)$ are subcomplexes, for each critical point $p$ and each simplex $\sigma \in T_{1}$.

Lemma 3.1 The cells in $T_{1}$ and $T_{0}$, together with all the cells $\mathcal{D}_{0}(p)$ and $\mathcal{F}(\sigma)$, give a legitimate cell decomposition, $T_{Y}^{\prime}$, of $Y$.

Proof Recall that $\mathcal{D}_{0}(p)$ and $\mathcal{F}(\sigma)$ have natural compactifications using broken flow lines (cf [7]). It may be shown by "induction on height" that these compactifications are homeomorphic to closed balls. There are moreover natural continuous maps of the compactifications to $Y$ which send the interiors of the balls homeomorphically to $\mathcal{D}_{0}(p)$ and $\mathcal{F}(\sigma)$. The transversality condition on $T_{1}$ and Assumption 1.1(b) ensure that the boundary of a cell consists of lower dimensional cells in $T_{Y}^{\prime}$.

We would like to glue the boundary components of $Y$ back together to obtain a nice cell decomposition of $X$, but usually $T_{0}$ will not agree with $T_{1}$. To correct for this, let $\rho:\left(\Sigma_{0}, T_{0}\right) \rightarrow\left(\Sigma_{1}, T_{1}\right)$ be a cellular approximation to the canonical identification $\Sigma_{0} \rightarrow \Sigma_{1}$. Consider the mapping cylinder of $\rho$ :

$$
M_{\rho}=\frac{\left(\Sigma_{0} \times[0,1]\right) \sqcup \Sigma_{1}}{(x, 1) \sim \rho(x)} .
$$

This has a cell decomposition consisting of $T_{0}$ and $T_{1}$, together with the cells $\Delta \times(0,1)$ for each $\Delta \in T_{0}$. There is a canonical inclusion $\Sigma_{0} \rightarrow M_{\rho}$ sending $x \mapsto(x, 0)$, and there is also a canonical inclusion $\Sigma_{1} \rightarrow M_{\rho}$. 
Definition 3.2 Let $X^{\prime}$ be the space obtained by gluing $Y$ and $M_{\rho}$ along $\Sigma_{0} \sqcup \Sigma_{1}$.

The space $X^{\prime}$ inherits a cell decomposition, but for our computations we prefer a simpler cell decomposition, obtained by fusing some cells together as follows. If $\Delta$ is a cell in $Y$ of the form $\mathcal{D}_{0}(p)$ or $\mathcal{F}(\sigma)$, we define a corresponding cell in $X^{\prime}$ by

$$
\hat{\Delta}:=\Delta \cup\left(\left(\partial \Delta \cap \Sigma_{0}\right) \times[0,1)\right) .
$$

Here $\left(\partial \Delta \cap \Sigma_{0}\right) \times[0,1)$ indicates a subset of $M_{\rho}$.

Definition 3.3 Let $T^{\prime}$ be the cell decomposition of $X^{\prime}$ consisting of cells of the following types:

(a) $\widehat{\mathcal{D}_{0}(p)}$ for $p \in Y$ a critical point;

(b) simplices in $T_{1}$;

(c) $\widehat{\mathcal{F}(\sigma)}$ for $\sigma \in T_{1}$.

\section{2 $X$ and $X^{\prime}$ have the same Reidemeister torsion}

We now show that $X$ and $X^{\prime}$ have the same Reidemeister torsion, if the Euler structures are compatible in an appropriate sense.

We begin by noting that $H_{*}\left(X^{\prime}\right)=H_{*}(X)$, and $H_{*}\left(\tilde{X}^{\prime}\right)=H_{*}(\tilde{X})$ as $\mathbb{Z}\left[H_{1}\right]-$ modules, as one can see from the exact sequences $(8),(9)$ below. Note that the universal abelian cover $\tilde{X}^{\prime}$ of $X^{\prime}$ is obtained from $\tilde{X}$ by modifying a neighborhood of the inverse image of $\Sigma$.

Notation 3.4 (1) If $Z$ is a subset of $X$ or $X^{\prime}$, then $\tilde{Z}$ will denote the inverse image of $Z$ in $\tilde{X}$ or $\tilde{X}^{\prime}$. So $\tilde{Z}$ is usually not the universal abelian cover of $Z$.

(2) We omit the cell structures from the notation when they are clear from context.

A smooth Euler structure $\xi$ on $X$ corresponds to an equivalence class of lifts of the critical points of $f$ to $\tilde{X}$, as in section 2.3.2. A combinatorial Euler structure $\xi^{\prime}$ on $X^{\prime}$ consists of an equivalence class of lifts of the cells $T^{\prime}$ to $\tilde{X}^{\prime}$.

Definition 3.5 We say that $\xi$ and $\xi^{\prime}$ are compatible if, within these equivalence classes, the lifts can be chosen so that: 
(a) The lift of each critical point $p$ in $\xi$ is contained in the lift of the cell $\widehat{\mathcal{D}_{0}(p)}$ in $\xi^{\prime}$.

(b) For each simplex $\sigma \in T_{1}$, the lift of $\sigma$ in $\xi^{\prime}$ agrees with the "top" of the lift of $\widehat{\mathcal{F}(\sigma)}$ in $\xi^{\prime}$.

The compatibility conditions in Definition 3.5 induce an isomorphism from $\operatorname{Eul}(X)$ to $\operatorname{Eul}\left(X^{\prime}, T^{\prime}\right)$ as affine spaces over $H_{1}$.

Recall that $\tau(X):= \pm \tau(X, o)$; similarly write $\tau\left(X^{\prime}, T^{\prime}\right):= \pm \tau\left(X^{\prime}, T^{\prime}, o\right)$.

Lemma 3.6 If the Euler structures $\xi \in \operatorname{Eul}(X)$ and $\xi^{\prime} \in \operatorname{Eul}\left(X^{\prime}, T^{\prime}\right)$ are compatible as above, then

$$
\tau(X)(\xi)= \pm \tau\left(X^{\prime}, T^{\prime}\right)\left(\xi^{\prime}\right)
$$

Proof The strategy is to compute the torsion of $X$ and $X^{\prime}$ by cutting them into pieces and using the product formula (Lemma 2.10) applied to various exact sequences, and see that we obtain the same answer. We proceed in three steps.

Step 1 Consider the cell decomposition on $\tilde{\Sigma} \times[0,1]$ consisting of cells $\tilde{\Delta} \times\{0\}$, $\tilde{\Delta} \times(0,1)$, and $\tilde{\Delta} \times\{1\}$, where $\tilde{\Delta}$ is a lift of a simplex $\Delta \in T_{1}$. Also recall that $\tilde{M}_{\rho}$ has a natural cell decomposition. We claim that with respect to these cell structures,

$$
\hat{\tau}\left(C_{*}(\tilde{\Sigma} \times[0,1]), e_{\Sigma}, h\right)= \pm \hat{\tau}\left(C_{*}\left(\tilde{M}_{\rho}\right), e_{\Sigma}^{\prime}, h^{\prime}\right),
$$

provided that the bases $e_{\Sigma}, h, e_{\Sigma}^{\prime}, h^{\prime}$ satisfy the following conditions:

(a) The bases $h, h^{\prime}$ for homology agree under the isomorphism induced by the canonical map $\Sigma \times[0,1] \rightarrow M_{\rho}$.

(b) The bases $e_{\Sigma}, e_{\Sigma}^{\prime}$ are given by lifts of cells such that:

(i) The lifts of the cells in $\Sigma \times\{1\} \subset \Sigma \times[0,1]$ and $\Sigma_{1} \subset M_{\rho}$ agree.

(ii) The lift of each cell $\Delta$ in $\Sigma \times\{0\} \subset \Sigma \times[0,1]$ or in $\Sigma_{0} \times\{0\} \subset M_{\rho}$ is adjacent to the lift of the cell $\Delta \times(0,1)$ in $\Sigma \times[0,1]$ or $M_{\rho}$ respectively.

To prove (6), we compute both sides by applying the product formula for torsion to the relative exact sequences

$$
\begin{gathered}
0 \rightarrow C_{*}(\tilde{\Sigma} \times\{1\}) \rightarrow C_{*}(\tilde{\Sigma} \times[0,1]) \rightarrow C_{*}(\tilde{\Sigma} \times[0,1], \tilde{\Sigma} \times\{1\}) \rightarrow 0, \\
0 \rightarrow C_{*}\left(\tilde{\Sigma}_{1}\right) \rightarrow C_{*}\left(\tilde{M}_{\rho}\right) \rightarrow C_{*}\left(\tilde{M}_{\rho}, \tilde{\Sigma}_{1}\right) \rightarrow 0 .
\end{gathered}
$$


The answers agree, because condition (a) implies that the $\tau\left(L_{*}\right)$ factors agree, condition (b(i)) implies that the $\hat{\tau}\left(C_{*}(\tilde{\Sigma})\right)$ factors agree, and condition (b(ii)) implies that

$$
\hat{\tau}\left(C_{*}(\tilde{\Sigma} \times[0,1], \tilde{\Sigma} \times\{1\}), e_{\Sigma}, 1\right)= \pm \hat{\tau}\left(C_{*}\left(\tilde{M}_{\rho}, \tilde{\Sigma}_{1}\right), e_{\Sigma}^{\prime}, 1\right)= \pm 1 .
$$

Step 2 Let $T_{Y}$ be a smooth triangulation on $Y$ whose restriction to each component of $\partial Y=\Sigma_{0} \sqcup \Sigma_{1}$ agrees with $T_{1}$. The smooth Euler structure $\xi$ on $X$ determines an equivalence class of bases, $e_{Y}$, for $C_{*}\left(\tilde{Y}, \tilde{\Sigma}_{0} ; T_{Y}\right)$, because $T_{Y}$ glues to a smooth triangulation of $X$.

Let $T_{Y}^{\prime}$ denote the cell decomposition of $Y$ given by Lemma 3.1. The combinatorial Euler structure $\xi^{\prime}$ on $X^{\prime}$ determines an equivalence class of bases $e_{Y}^{\prime}$ for $C_{*}\left(\tilde{Y}, \tilde{\Sigma_{0}} ; T_{Y}^{\prime}\right)$, because the cells of $T_{Y}^{\prime}$ in $Y \backslash \Sigma_{0}$ are in one to one correspondence with the cells of $X^{\prime}$.

We claim that

$$
\hat{\tau}\left(C_{*}\left(\tilde{Y}, \tilde{\Sigma_{0}} ; T_{Y}^{\prime}\right), e_{Y}^{\prime}, h^{\prime}\right)= \pm \hat{\tau}\left(C_{*}\left(\tilde{Y}, \tilde{\Sigma_{0}} ; T_{Y}\right), e_{Y}, h\right)
$$

provided that the bases $h^{\prime}$ and $h$ on homology agree.

To prove (7), note that the pullback of the Morse function $f$ to $Y$ lifts to a real-valued function $\hat{f}: Y \rightarrow \mathbb{R}$. Let $C M_{*}(\hat{f})$ denote the Morse complex of $\hat{f}$ on the covering $\tilde{Y}$. A direct computation, using the compatibility of $\xi$ and $\xi^{\prime}$, shows that

$$
\hat{\tau}\left(C_{*}\left(\tilde{Y}, \tilde{\Sigma_{0}} ; T_{Y}^{\prime}\right), e_{Y}^{\prime}, h^{\prime}\right)= \pm \hat{\tau}\left(C M_{*}(\hat{f}), e^{\prime \prime}, h^{\prime \prime}\right) .
$$

(For similar calculations see section 3.3 and section 3.4; the result here corresponds essentially to setting $t=0$ in (11) and Lemma 3.7.) Here the basis $e^{\prime \prime}$ for $C M_{*}(\hat{f})$ is determined by the lifts of the critical points determined by $\xi$ as before, and we assume that the bases $h^{\prime}, h^{\prime \prime}$ on homology agree under the standard isomorphism $H_{*}\left(\tilde{Y}, \tilde{\Sigma_{0}}\right) \simeq H_{*}\left(C M_{*}(\hat{f})\right)$.

We also have

$$
\hat{\tau}\left(C M_{*}(\hat{f}), e^{\prime \prime}, h^{\prime \prime}\right)= \pm \hat{\tau}\left(C_{*}\left(\tilde{Y}, \tilde{\Sigma_{0}} ; T_{Y}\right), e_{Y}, h\right) .
$$

The idea of the proof is to vary $\hat{f}$ in the space of Morse functions on $Y$ such that (i) the gradient points outward along $\Sigma_{0}$ and does not point outward along $\Sigma_{1}$, and (ii) wherever the gradient is tangent to $\Sigma_{1}$, the inward covariant derivative of the gradient points inward. As in section 2.4, one can show that the resulting torsion is independent of the Morse function. Deforming $\hat{f}$ to a Morse function $F_{Y}$ adapted to the triangulation $T_{Y}$, such that $C M_{*}\left(F_{Y}\right)=C_{*}\left(\tilde{Y}, \tilde{\Sigma}_{0}, T_{Y}\right)$, we have $\hat{\tau}\left(C M_{*}(\hat{f}), e^{\prime \prime}, h^{\prime \prime}\right)= \pm \hat{\tau}\left(C M_{*}\left(F_{Y}\right), i_{f F}\left(e^{\prime \prime}\right), h^{\prime \prime}\right)$, where $i_{f F}\left(e^{\prime \prime}\right)$ is the 
equivalence class of lifts (which correspond to bases of $C M_{*}$ ) induced from $e^{\prime \prime}$ via the homotopy from $\hat{f}$ to $F_{Y}$. But $i_{f F}\left(e^{\prime \prime}\right)=e_{Y}$ because the homotopy from $\nabla \hat{f}$ to $\nabla F_{Y}$ extends to a homotopy of vector fields on $X=Y \cup(\Sigma \times I)$, which is homotopic to a homotopy from $\nabla f$ to the standard vector field associated to the triangulation of $X$ ( $\mathrm{cf}$ end of section 2.2).

The above two equations prove (7).

Step 3 We now use (6) and (7) to compute the torsion of $X$ and $X^{\prime}$.

We can regard $X$ as the union of $Y$ and $\Sigma \times[0,1]$ along $\Sigma_{0} \sqcup \Sigma_{1}$. Let $\bar{T}$ denote the cell decomposition of $X$ obtained by gluing the triangulation $T_{Y}$ of $Y$ to the product cell structure on $\Sigma \times[0,1]$ obtained from $T_{1}$. We then have a short exact sequence

$$
0 \rightarrow C_{*}\left(\tilde{\Sigma_{0}} \sqcup \tilde{\Sigma_{1}}\right) \rightarrow C_{*}(\tilde{\Sigma} \times[0,1]) \oplus C_{*}\left(\tilde{Y} ; T_{Y}\right) \rightarrow C_{*}(\tilde{X} ; \bar{T}) \rightarrow 0 .
$$

Let $\overline{T^{\prime}}$ denote the "unfused" cell decomposition of $X^{\prime}$ from section 3.1. We then have a short exact sequence

$$
0 \rightarrow C_{*}\left(\tilde{\Sigma_{0}} \sqcup \tilde{\Sigma_{1}}\right) \rightarrow C_{*}\left(\tilde{M}_{\rho}\right) \oplus C_{*}\left(\tilde{Y} ; T_{Y}^{\prime}\right) \rightarrow C_{*}\left(\tilde{X^{\prime}} ; \overline{T^{\prime}}\right) \rightarrow 0 .
$$

We can choose representatives $e_{Y}, e_{Y}^{\prime}$ such that they agree on $\Sigma \times\{1\}$ and $\Sigma_{1}$ with $e_{\Sigma}, e_{\Sigma}^{\prime}$ respectively. Let $e(\xi)$ denote the basis for $C_{*}(\tilde{X} ; \bar{T})$ obtained by combining the bases $e_{\Sigma}, e_{Y}$ of Step 1 and Step 2 respectively. Similarly let $e^{\prime}\left(\xi^{\prime}\right)$ denote the basis for $C_{*}\left(\tilde{X}^{\prime} ; \overline{T^{\prime}}\right)$ obtained by combining the bases $e_{\Sigma}^{\prime}, e_{Y}^{\prime}$ of Step 1 and Step 2. Then $e(\xi)$ is a representative of the combinatorial Euler structure on $(X, \bar{T})$ corresponding to the smooth Euler structure $\xi$, and $e^{\prime}\left(\xi^{\prime}\right)$ is a representative of the image of $\xi^{\prime}$ under the canonical isomorphism $\operatorname{Eul}\left(X^{\prime}, T^{\prime}\right) \rightarrow \operatorname{Eul}\left(X^{\prime}, \bar{T}^{\prime}\right)$.

Applying the product formula to the above exact sequences, and using equations (6) and (7), we obtain

$$
\hat{\tau}\left(C_{*}(\tilde{X} ; \bar{T}), e(\xi), h\right)= \pm \hat{\tau}\left(C_{*}\left(\tilde{X}^{\prime} ; \overline{T^{\prime}}\right), e^{\prime}\left(\xi^{\prime}\right), h^{\prime}\right) .
$$

Here we are assuming that the bases $h, h^{\prime}$ for homology agree under the natural isomorphism $H_{*}(\tilde{X}) \simeq H_{*}\left(\tilde{X}^{\prime}\right)$. Also, to apply (7) in the above computation, one relates $\tilde{Y}$ to the pair $\left(\tilde{Y}, \Sigma_{0}\right)$ as in Step 1 .

In particular, equation (10) implies that

$$
\tau\left(C_{*}(\tilde{X} ; \bar{T}), e(\xi)\right)= \pm \tau\left(C_{*}\left(\tilde{X}^{\prime} ; \overline{T^{\prime}}\right), e^{\prime}\left(\xi^{\prime}\right)\right) .
$$

This implies the lemma because $\tau\left(C_{*}(\tilde{X} ; \bar{T}), e(\xi)\right)=\tau(X)(\xi)$, since the insertion of $\Sigma \times[0,1]$ changes nothing, and similarly $\tau\left(C_{*}\left(\tilde{X}^{\prime} ; \overline{T^{\prime}}\right), e^{\prime}\left(\xi^{\prime}\right)\right)=$ $\tau\left(X^{\prime}, T^{\prime}\right)\left(\xi^{\prime}\right)$. 


\subsection{Computing the torsion}

We now compute the torsion of the approximating cell complex $\left(X^{\prime}, T^{\prime}\right)$, for a combinatorial Euler structure $\xi^{\prime}$ compatible with a smooth Euler structure $\xi$ on $X$ as in the previous section.

Since Lemma 2.13 proves the theorem for real-valued Morse functions, we assume from now on that

$$
\theta \neq 0 \text {. }
$$

Without loss of generality, we may also assume that $\theta$ is indivisible in $H^{1}(X ; \mathbb{Z})$. (If $\theta$ is divisible by $k$, we can lift $f$ to a $k$-fold cover of $S^{1}$ without changing the invariant $I$.) Let $V:=\operatorname{Ker}(\theta)$, and choose a splitting

$$
H_{1}(X)=V \oplus \mathbb{Z} \text {. }
$$

Let $t$ denote the generator of the $\mathbb{Z}$ component with $\theta(t)=-1$. Then the Novikov ring can be identified with the ring of formal Laurent series in $t$ with coefficients in $\mathbb{Z}[V]$ :

$$
\Lambda=\mathbb{Z}[V]((t)) .
$$

Recall that $Q(\Lambda)$ is a finite sum of fields. To prove Theorem 1.7, it suffices to show that it holds after projecting to each such field. Let $K$ be a field component of $Q(\Lambda)$. By the Novikov isomorphism (Theorem 2.1), the complexes $C N_{*} \otimes K$ and $C_{*}(\tilde{X}) \otimes K$ have isomorphic homology. So we will assume that these complexes are both acyclic, since otherwise they both have zero torsion $\tau$, and there is nothing to prove. In all of the calculations below, we implicitly tensor everything with the field $K$.

We can decompose

$$
C_{i}\left(\tilde{X}^{\prime}\right)=D_{i} \oplus E_{i} \oplus F_{i}
$$

where the three summands are generated by the cells of types (a), (b), and (c) respectively from Definition 3.3. Let us choose a basis $e\left(\xi^{\prime}\right)$ for $C_{i}\left(\tilde{X}^{\prime}\right)$ as in Definition 3.5. We can identify

$$
F_{i} \simeq E_{i-1} .
$$

The matrix for the boundary operator on $C_{i}\left(\tilde{X}^{\prime}\right)$ can then be written as

$$
\partial_{i}=\begin{array}{ccc}
D_{i} & E_{i} & F_{i} \\
D_{i-1} \\
E_{i-1} \\
F_{i-1}
\end{array}\left(\begin{array}{ccc}
\mathbf{N}_{i} & 0 & \mathbf{W}_{i} \\
-t \mathbf{M}_{i} & \partial_{i}^{\Sigma} & 1-t \phi_{i-1} \\
0 & 0 & -\partial_{i-1}^{\Sigma}
\end{array}\right) .
$$


We remark that $\partial_{i}^{\Sigma}$ is the boundary operator on $C_{*}(\tilde{\Sigma})$. Also $\phi_{i-1}$ is a matrix with entries in $\mathbb{Z}[V]$, which can be interpreted as the return map of the gradient flow from $\tilde{\Sigma}$ to $\tilde{\Sigma}$, after perturbation by our cellular approximation $\rho$. Likewise $\mathbf{M}_{i}$ sends $\widehat{\mathcal{D}_{0}(p)}$, where $p \in \tilde{X}$ is a critical point, to a perturbation of the "descending slice" $\widehat{\mathcal{D}_{0}(p)} \cap \tilde{\Sigma}$.

Continuing the calculation, due to the acyclicity assumption we may choose decompositions $D_{i}=D_{i}^{A} \oplus D_{i}^{B}$ such that $D_{i}^{A}$ and $D_{i}^{B}$ are spanned by (cells corresponding to) critical points, and the differential $\partial^{f}$ induces an isomorphism $D_{i}^{A} \rightarrow D_{i-1}^{B}$. In the notation below, we denote matrices with domain or range $D_{*}$ by boldface letters, and we denote their restrictions to $D_{i}^{A}$ and/or projections to $D_{i-1}^{B}$ by plain letters.

We now apply Lemma 2.7 with $A_{i}=D_{i}^{A} \oplus F_{i}$ and $B_{i}=D_{i}^{B} \oplus E_{i}$. (We will explain in a moment why this choice of $A_{i}$ and $B_{i}$ is legitimate.) We obtain

$$
\tau\left(C_{*}\left(\tilde{X}^{\prime}, e\left(\xi^{\prime}\right)\right)=\prod_{i=1}^{n} \operatorname{det}\left(\Omega_{i}\right)^{(-1)^{i+1}}\right.
$$

where

$$
\Omega_{i}=\begin{gathered}
D_{i}^{A} \\
D_{i-1}^{B} \\
E_{i-1}
\end{gathered}\left(\begin{array}{cc}
N_{i} & W_{i} \\
-t M_{i} & 1-t \phi_{i-1}
\end{array}\right) .
$$

We note that $1-t \phi_{i-1}$ is invertible because $\phi_{i-1}$ has entries in $\mathbb{Z}[V]$. It follows that

$$
\operatorname{det}\left(\Omega_{i}\right)=\operatorname{det}\left(1-t \phi_{i-1}\right) \operatorname{det}\left(K_{i}\right)
$$

where

$$
\mathbf{K}_{i}:=\mathbf{N}_{i}+t \mathbf{W}_{i}\left(1-t \phi_{i-1}\right)^{-1} \mathbf{M}_{i}: D_{i} \rightarrow D_{i-1} .
$$

It will follow from Lemma $3.9(\mathrm{~b})$ and the choice of $D_{i}^{A}, D_{i}^{B}$ that $K_{i}$ is nonsingular, provided that the triangulation $T_{1}$ is sufficiently fine and the cellular approximation $\rho$ is sufficiently close to the identity. In particular, the matrices $\Omega_{i}$ are then nonsingular, so that Lemma 2.7 legitimately applies to the $A_{i}$ and $B_{i}$ chosen above.

In conclusion, the above calculations imply the following lemma.

Lemma 3.7 If $T_{1}$ is sufficiently fine and $\rho$ is sufficiently close to the identity, then

$$
\tau\left(C_{*}\left(\tilde{X}^{\prime}\right)\right)\left(\xi^{\prime}\right)=\prod_{i=1}^{n}\left(\operatorname{det}\left(1-t \phi_{i-1}\right) \operatorname{det}\left(K_{i}\right)\right)^{(-1)^{i+1}} .
$$




\subsection{Geometric interpretation}

We will now interpret the factors on the right side of Lemma 3.7 in terms of Morse theory.

Notation 3.8 Suppose $x, y$ are elements of $\Lambda=\mathbb{Z}[V]((t))$, or matrices with entries in $\Lambda$, which might depend on the choice of triangulation $T_{1}$ and cellular approximation $\rho$. We write

$$
x \approx y
$$

if $x-y=O\left(t^{k}\right)$, where $k$ can be made arbitrarily large by choosing $T_{1}$ sufficiently fine and $\rho$ sufficiently close to the identity.

Lemma 3.9 (a) $\prod_{i=0}^{n-1} \operatorname{det}\left(1-t \phi_{i}\right)^{(-1)^{i}} \approx \zeta$.

(b) Under the natural identification $D_{*} \simeq C N_{*}$, we have

$$
\mathbf{K}_{i} \approx \partial_{i}^{f} \text {. }
$$

Proof (a) Let $\hat{f}: \tilde{X} \rightarrow \mathbb{R}$ be a lift of $f$, and let $\tilde{\Sigma}:=\hat{f}^{-1}(0)$. The downward gradient flow of $\hat{f}$ induces partially defined return maps

$$
\varphi^{k}: \tilde{\Sigma} \rightarrow t^{k} \tilde{\Sigma} .
$$

The definition (1) of $\zeta$ is equivalent to

$$
\zeta=\exp \left(\sum_{k>0, g \in V} \operatorname{Fix}\left(\varphi^{k} \circ t^{-k} g^{-1}\right) \frac{g t^{k}}{k}\right) \in \mathbb{Z}[V]((t))=\Lambda .
$$

Here $\operatorname{Fix}(s)$ counts fixed points of the equivariant map $s$ modulo covering transformations, with their Lefschetz signs.

Suppose to begin that $\rho=\mathrm{id}$. By the machinery used to prove the Lefschetz fixed point theorem in [1], for each $k$ we have

$$
\sum_{g \in V} \operatorname{Fix}\left(\varphi^{k} \circ t^{-k} g^{-1}\right) \cdot g=\sum_{i=0}^{n-1}(-1)^{i} \operatorname{Tr}\left(\phi_{i}^{k}\right) \in \mathbb{Z}[V] .
$$

In this case we have

$$
\zeta=\prod_{i=0}^{n-1} \operatorname{det}\left(1-t \phi_{i}\right)^{(-1)^{i}} .
$$

To see this, it is enough to check that the logarithmic derivatives of both sides are equal, which follows from equations (13) and (14). 
In general, let $H: \Sigma \times[0,1] \rightarrow \Sigma$ be the homotopy from id to $\rho$. In [7] we defined a natural compactification $\bar{\Gamma} \subset \Sigma \times \Sigma$ of the graph of $\varphi$. Using this one can define a compactified graph $\bar{\Gamma}_{t}^{i}$ of $\left.(H(\cdot, t) \circ \varphi)^{i}\right)$ in a similar manner. Now there exists a positive integer $N$ such that if the cells in $T_{1}$ are all contained in balls of radius $\epsilon$, then the homotopy $H$ can be chosen so that $\operatorname{dist}(H(t, x), x)<N \epsilon$ for all $t \in[0,1]$ and $x \in \Sigma$. (Cf the construction of $H$ in [23]; by carefully controlling each intermediate step in the homotopy, the above claim may be achieved.) Also, the set of fixed points of $\varphi^{i}$ lies in the interior of $\bar{\Gamma}^{i}$ under the diagonal map $\Sigma \rightarrow \Sigma \times \Sigma$ by definition of $\bar{\Gamma}^{i}$, and is compact as a consequence of Assumption 1.1. It follows that for any positive integer $k$ we can choose $\epsilon$ so that for all $i \leq k$ and all $t \in[0,1]$, the compactified graph $\bar{\Gamma}_{t}^{i}$ does not cross the diagonal in $\Sigma \times \Sigma$. Then equation (14) will hold up to order $k$, and therefore so will equation (15).

(b) If $p$ is a critical point of index $i$, then

$$
\partial[\mathcal{D}(p)]=\left[\mathcal{D}\left(\partial^{f} p\right)\right]
$$

where the brackets indicate the fundamental class of the compactification of the descending manifold [7]. Now suppose again that $\rho=\mathrm{id}$. Recall from equation (12) that the matrix $\mathbf{K}_{i}$ sends $\widehat{\mathcal{D}_{0}(p)}$ to a linear combination of cells of the form $\widehat{\mathcal{D}_{0}(q)}$, where $q$ is a critical point of index $i-1$. (We will henceforth omit the hats ${ }^{\wedge}$ on $\mathcal{D}_{0}$ or $\mathcal{F}$ when $\rho=$ id, since in this case hatted and unhatted versions can be identified.) In fact,

$$
\mathbf{K}_{i}\left(\mathcal{D}_{0}(p)\right)=\mathcal{D}_{0}\left(\partial^{f}(p)\right) .
$$

To see this, note that from the definition of $\mathbf{M}_{i}$ in equation (11), we have

$$
[\mathcal{D}(p)]=\mathcal{D}_{0}(p)+\sum_{k=0}^{\infty} t^{k+1} \mathcal{F}\left(\phi^{k} \mathbf{M}_{i}\left(\mathcal{D}_{0}(p)\right)\right) .
$$

(Here the initial descending manifolds $\mathcal{D}_{0}$ and initial downward flow $\mathcal{F}$ are defined as in section 3.1, but using $\tilde{X}$ and $\tilde{\Sigma}$ instead of $X$ and $\Sigma$.) Applying equation (11) to this gives

$$
\partial[\mathcal{D}(p)]=\mathbf{K}_{i}\left(\mathcal{D}_{0}(p)\right)+(\text { terms without initial descending manifolds }) .
$$

Equations (16) and (17) imply that $\partial_{i}^{f}=\mathbf{K}_{i}$ when $D_{*}$ (the domain/range of $\left.\mathbf{K}_{*}\right)$ is identified with $C N_{*}$.

The case $\rho \neq$ id can be handled similarly to part (a). 
We can now complete the proof of Theorem 1.7. Lemmas 3.9(b) and 2.7 imply that

$$
\left.\prod_{i=1}^{n} \operatorname{det}\left(K_{i}\right)\right)^{(-1)^{i+1}} \approx \tau\left(C N_{*}\right)(\xi) .
$$

Together with Lemmas 3.6, 3.7, and 3.9(a), this implies that Theorem 1.7 holds up to order $k$ for all $k$.

\section{The 3-dimensional case and Seiberg-Witten the- ory}

We now review from [7] the definition of the Morse-theoretic invariant $I_{3}$, and the background for Conjecture 1.9 relating this invariant to Seiberg-Witten theory. We will then prove Theorem 1.12, relating this invariant to Turaev torsion.

\subsection{Motivation from Seiberg-Witten theory}

Let $X$ be a closed connected oriented smooth $3-$ manifold with $b_{1}(X)>0$. Let $\mathfrak{s}$ be a spin-c structure on $X$. This determines a $U(2)$-bundle $S \rightarrow X$ with a Clifford action of $T X$ on $S$. A section $\psi$ of $S$ and a connection $A$ on $\operatorname{det}(S)$ satisfy the Seiberg-Witten equations with perturbation $\omega$ if, in the notation of $[9]$,

$$
\begin{aligned}
\not \partial_{A} \psi & =0, \\
\rho\left(F_{A}\right) & =i \sigma(\psi, \psi)+i \rho(\omega) .
\end{aligned}
$$

The Seiberg-Witten invariant SW(s) counts solutions to these equations modulo gauge equivalence. (For more on 3-dimensional Seiberg-Witten invariants see eg $[13,14,18]$.)

Let us choose the perturbation to be $\omega=r * d f$, where $f: X \rightarrow S^{1}$ is harmonic, * denotes the Hodge star, and $r$ is a real number. By perturbing the metric, we may arrange that $f$ is a Morse function. Away from the critical points, the spinor bundle $S$ splits into eigenspaces of Clifford multiplication by $d f$,

$$
S=E \oplus\left(E \otimes K^{-1}\right),
$$

where $K^{-1}:=\operatorname{Ker}(d f: T X \rightarrow \mathbb{R})$. 
Taking $r \rightarrow \infty$, one expects that for a Seiberg-Witten solution the zero set of the $E$ component of $\psi$ to become parallel to $\nabla f$. (The energy of the SeibergWitten solution will be concentrated along this zero set. For detailed analysis see [24] and its sequels.) This suggests that $\mathrm{SW}(\mathfrak{s})$ counts unions of closed orbits and flow lines of $\nabla f$ starting and ending at critical points, whose total homology class is Poincaré dual to $c_{1}(E)$.

The above homological condition implies that in our union of closed orbits and flow lines there is precisely one flow line starting at each index 2 critical point and ending at each index 1 critical point. (See [7]. There are no index 0 or 3 critical points because $f$ is harmonic, and there are equally many index 1 and index 2 points because $\chi(X)=0$.) In other words, in the notation of section 2.2 , our union of closed orbits and flow lines lives in $H_{1}(X, v)$, where $v=-\nabla f$. As in Remark 1.10, the counting of closed orbits is related to Taubes' counting of pseudoholomorphic tori in symplectic 4-manifolds [25], which indicates that we should allow closed orbits to be multiply covered when they are elliptic, but not when they are hyperbolic.

\subsection{The definition of $I_{3}$}

We now want to define $I_{3}(\mathfrak{s})$ to be a signed count of such unions of closed orbits and flow lines. A convenient way to do so is to use generating functions as follows. Choose orderings of the index 1 and index 2 critical points. Let $\mathcal{P}^{i j}$ denote the set of flow lines from the $i^{\text {th }}$ index 2 point to the $j^{\text {th }}$ index 1 point. Define the path matrix $P$ by

$$
P^{i j}:=\sum_{\gamma \in \mathcal{P}^{i j}} \epsilon(\gamma)[\gamma]
$$

Here $[\gamma] \in H_{1}\left(X, v^{-1}(0)\right)$ is the homology class of $\gamma$ (oriented downward), and $\epsilon(\gamma)$ is the sign of $\gamma$ as in section 2.1. The entries of $P$ live in the Novikov ring of the relative homology group $H_{1}\left(X, v^{-1}(0)\right)$ with grading given by minus intersection number with $\Sigma$.

Note that $\operatorname{det}(P)$, regarded as a $\mathbb{Z}$-valued function on $H_{1}\left(X, v^{-1}(0)\right)$, is supported on $H_{1}(X, v)$. Also, the subset of $\operatorname{Nov}\left(H_{1}\left(X, v^{-1}(0)\right)\right)$ consisting of functions supported on $H_{1}(X, v)$ is a $\Lambda$-submodule. A generating function counting unions of closed orbits and flow lines of the type we want is now given by

$$
I_{3}^{v}:=\zeta \cdot \operatorname{det}(P) \in \operatorname{Nov}\left(H_{1}(X, v)\right) .
$$

In the above equation, ' ' denotes the $\Lambda$ action. The closed orbits are counted correctly as a result of the product formula for the zeta function (2). 
Let $j_{v}: \operatorname{Spin}^{c}(X) \rightarrow H_{1}(X, v)$ denote the map that sends a spin-c structure to the Poincaré-Lefschetz dual of $c_{1}(E)$, where $E$ is the line bundle defined in (18). The map $j_{v}$ is an $H_{1}$-equivariant isomorphism.

Definition $4.1[7]$ Regarding $I_{3}^{v}$ as a function $H_{1}(X, v) \rightarrow \mathbb{Z}$, we define

$$
I_{3}:=I_{3}^{v} \circ j_{v}: \operatorname{Spin}^{c}(X) \rightarrow \mathbb{Z} .
$$

This definition makes sense for any Morse function $f: X \rightarrow S^{1}$ with no index 0 or 3 critical points, even if $f$ is not harmonic. The calculation below will show that $I_{3}$ does not depend on $f$, except that there is a global sign ambiguity in $I_{3}$ due to the orientation choices we made. (Also $I_{3}$ depends on the sign of $\theta \in H^{1}(X ; \mathbb{Z})$ when $b_{1}(X)=1$.)

\subsection{Relation with Turaev torsion}

To relate $I_{3}$ to torsion, we note that the isomorphism $i_{v} \circ j_{v}: \operatorname{Spin}^{c}(X) \rightarrow$ $\operatorname{Eul}(X)$ does not depend on $v$. It follows that there is a canonical isomorphism $\iota: \operatorname{Spin}^{c}(X) \rightarrow \operatorname{Eul}(X)$. (This isomorphism was first defined by Turaev [27] in a different but equivalent way. The inverse map sends a smooth Euler structure represented by a nonsingular vector field $u$ to the spin-c structure whose spin bundle is $\mathbb{C} u \oplus u^{\perp}$ with a standard Clifford action.) In summary, we have the following commutative triangle:

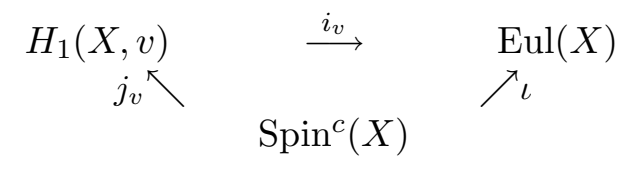

Proof of Theorem 1.12 It is enough to show that

$$
I_{3}^{v} \circ i_{v}^{-1}= \pm T(X ; o): \operatorname{Eul}(X) \rightarrow \mathbb{Z} .
$$

By Theorem 1.7 and the definition of $T$, this is equivalent to asserting that for some orientation choice, we have

$$
\operatorname{det}(P)\left(i_{v}^{-1}(\xi)\right)=\tau\left(C N_{*}\right)(\xi)(0)
$$

for all Euler structures $\xi$. If $\xi_{0}$ is a reference Euler structure and $\gamma:=i_{v}^{-1}\left(\xi_{0}\right) \in$ $H_{1}(X, v)$, then this equation is equivalent to

$$
\operatorname{det}(P)(\cdot+\gamma)=\tau\left(C N_{*}\right)\left(\xi_{0}\right)(\cdot): H_{1} \rightarrow \mathbb{Z} .
$$

This last equation follows from the definition of $\tau\left(C N_{*}\right)$ and Example 2.9. 


\section{References}

[1] R F Brown, The Lefschetz fixed point theorem, Scott, Foresman and Co. (1971)

[2] D Fried, Homological identities for closed orbits, Invent. Math. 71 (1983) 419442

[3] D Fried, Lefschetz formulas for flows, from: "The Lefschetz centennial conference, part III", Contemp. Math. 58, III, Amer. Math. Soc. Providence, R.I. (1987) 19-69

[4] K Fukaya, The symplectic s-cobordism conjecture: a summary, from: "Geometry and Physics (Aarhus, 1995)", Lecture Notes in Pure and Appl. Math. 184, Dekker (1997) 209-219

[5] H Hofer, D Salamon, Floer homology and Novikov rings, from: "The Floer memorial volume", Progr. Math. 133, Birkhäuser, Basel (1995) 483-524

[6] M Hutchings, Reidemeister torsion in generalized Morse theory, e-print math.DG/9907066

[7] M Hutchings, Y-J Lee, Circle-valued Morse theory, Reidemeister torsion, and Seiberg-Witten invariants of three-manifolds, Topology, 38 (1999) 861-888

[8] E Ionel, T Parker, Gromov invariants and symplectic maps, e-print dg-ga/9703013

[9] P B Kronheimer, T S Mrowka, The genus of embedded surfaces in the projective plane, Math. Res. Lett. (1994) 797-806

[10] F Latour, Existence de 1-formes fermées non singuliéres dans une classe de cohomologie de de Rham, Inst. Hautes Études Sci. Publ. Math. No. 80 (1994) 135-194

[11] F Laudenbach, On the Thom-Smale complex, appendix to J-M Bismut \& W Zhang, An extension of a theorem by Cheeger and Müller, Asterisque, 205 (1992)

[12] Y-J Lee, Morse theory and Seiberg-Witten monopoles on 3-manifolds, Harvard University thesis (1997)

[13] Y Lim, Seiberg-Witten theory of 3-manifolds, preprint (1996)

[14] G Meng, CH Taubes, $\underline{S W}=$ Milnor torsion, Math. Res. Lett. 3 (1996) 661-674

[15] J Milnor, Infinite cyclic coverings, Collected works vol. 2, Publish or Perish (1996)

[16] J Milnor, Whitehead torsion, Bull. Amer. Math. Soc. 72 (1966) 358-426

[17] S P Novikov, Multivalued functions and functionals. An analogue of the Morse theory, Soviet Math. Dokl. 24 (1981) 222-226

[18] C Okonek, A Teleman, 3-dimensional Seiberg-Witten invariants and nonKählerian geometry, Math. Ann. 312 (1998) 261-288 
[19] A Pazhitnov, On the Novikov complex for rational Morse forms, Ann. Fac. Sci. Toulouse Math. (6) 4 (1995) 297-338

[20] A Pajitnov, Simple homotopy type of Novikov complex for closed 1-forms and Lefschetz zeta functions of the gradient flow, eprint dg-ga/9706014

[21] M Pozniak, Floer homology, Novikov rings, and clean intersections, Univ. of Warwick thesis (1994)

[22] D Salamon, Seiberg-Witten invariants of mapping tori, symplectic fixed points, and Lefschetz numbers, from: "Proceedings of Gökova Geometry-Topology Conference 1998", (S Akbulut, T Önder and R Stern, editors), International Press (1999) 117-143

[23] E Spanier, Algebraic topology, Springer-Verlag corrected reprint (1989)

[24] C H Taubes, The Seiberg-Witten and Gromov invariants, Math. Res. Lett. 2 (1995) 221-238

[25] CH Taubes, Counting pseudo-holomorphic submanifolds in dimension 4, J. Differential Geom. 44 (1996) 818-893

[26] V Turaev, Reidemeister torsion in knot theory, Russian Math. Surveys 41 (1986) 119-182

[27] V Turaev, Euler structures, nonsingular vector fields, and torsions of Reidemeister type, Math. USSR-Izv. 34 (1990) 627-662

[28] V Turaev, Torsion invariants of $\mathrm{Spin}^{c}$ structures on 3-manifolds, Math. Res. Lett. 4 (1997) 679-695

[29] V Turaev, A combinatorial formulation for the Seiberg-Witten invariants of 3-manifolds, Math. Res. Lett. 5 (1998) 583-598

[30] A Weil, Numbers of solutions of equations in finite fields, Bull. Amer. Math. Soc. 55 (1949) 497-508 